\title{
The discrimination of acute tubular necrosis and prerenal azotemia using two biomarkers simultaneously
}

\author{
Abbas Ali Zeraati ${ }^{1}{ }^{\circledR}$, Farnaz Sahihi $^{1}{ }^{\circledR}$, Zahra Lotfi $^{{ }^{*}}{ }^{\circledR}$, Freshteh Mamdouhi $^{1}$, Farzaneh Sharifipour ${ }^{1}$, \\ Tina Zeraati ${ }^{1,2}$ \\ ${ }^{1}$ Kidney Transplantation Complications Research Center, Faculty of Medicine, Mashhad University of Medical Science, Mashhad, Iran \\ ${ }^{2}$ Research Committee, Mashhad University of Medical Science, Mashhad, Iran
}

\section{AR T I C L E I N F O}

Article Type:

Original

\section{Article History:}

Received: 9 August 2019

Accepted: 30 November 2019

Published online: 18 December 2019

\section{Keywords:}

Prerenal azotemia

Acute tubular necrosis

Lithium

Chronic kidney disease

\begin{abstract}
A B S T R A C T
Introduction: The fractional excretion of sodium (FE Na) in urine has appeared as a helpful way to distinguish prerenal azotemia from acute tubular necrosis (ATN).

Objectives: The urinary index of sodium has some limits. Lithium can be an additional careful indicator. The goal of our study was to assess the standards fractional excretion of sodium and lithium, (FE Na and FE Li) in distinguishing pre-renal azotemia (PRA) from ATN.

Patients and Methods: Twenty-seven patients with prerenal azotemia, 25 patients with ATN and 20 healthy persons were included in this investigation. The plasma and urine sodium, creatinine and lithium levels were assessed. Additionally, FE Na and FE Li were calculated. To assess the diagnostic usefulness of FE Na and FE Li in discriminating prerenal azotemia from ATN, we created a receiver operating characteristic (ROC) curve.

Results: The area under the curves (AUCs) of fractional excretion of Li and Na were 0.84 and 0.83 for distinguishing prerenal azotemia from ATN, respectively. There was a significant direct association between FE Na and FE Li in patients with ATN ( $\mathrm{P}=0.001)$. No significant association of FE Na and FE $\mathrm{Li}$ in patients with prerenal azotemia was detected $(\mathrm{p}=0.26)$. By a cutoff point of $2.96 \%$, the sensitivity and specificity for $\mathrm{FE} \mathrm{Na}$, were $68 \%$ and $75 \%$, respectively for distinguishing PRA from ATN. By a cutoff point of $4.17 \%$, the sensitivity and specificity of FE Li were $80 \%$ and $79 \%$, respectively, for distinguishing prerenal azotemia from ATN.

Conclusion: This investigation appeared a high AUC and accuracy of fractional excretion of sodium and lithium as a diagnostic method for distinguishing prerenal azotemia from ATN when used simultaneously. However, the discrimination of sensitivity and specificity of fractional excretion of lithium was greater than the fractional excretion of $\mathrm{Na}$.
\end{abstract}

\section{Implication for health policy/practice/research/medical education}

In a study on 27 patients with prerenal azotemia, 25 patients with acute tubular necrosis and 20 healthy persons, we found accuracy of fractional excretion of sodium and lithium as a diagnostic method for distinguishing prerenal azotemia from acute tubular necrosis when used simultaneously. However, the discrimination of sensitivity and specificity of fractional excretion of lithium was greater than the fractional excretion of $\mathrm{Na}$.

Please cite this paper as: Zeraati AA, Sahihi F, Lotfi Z, Sharifipour F, Zeraati T. The discrimination of acute tubular necrosis and prerenal azotemia using two biomarkers simultaneously. J Renal Inj Prev. 2020; 9(1): e03. DOI: 10.15171/jrip.2020.03.

\section{Introduction}

Acute kidney injury is a common clinical problem that its prevention and treatment is very important (1). The main causes of renal failure consist of pre-renal azotemia (PRA) and acute tubular necrosis (ATN). For proper treatment, the discrimination of these two diseases from each other is very crucial.

Considering the fact that the invasive diagnostic measures such as renal biopsy, and also urinary diagnostic modalities, may not have an acceptable value in the critical conditions, thus finding a reliable non-invasive diagnostic method is necessary (2).

On the other hand, if the treatment of acute renal failure is delayed, a possibility to progress to ATN was existed. Therefore, there is a necessity for a rapid and reliable differential diagnostic test to differentiate them. Various urinary indices have been proposed for the early diagnosis of acute renal failure, among them fractional excretion of sodium is a useful tool in the differentiation of PRA from ATN (2). However, the available urinary markers 
are limited, for example in the case of administration of diuretics or presence of diabetes (3). In such cases of sodium loss in patients with heart or kidney failure or liver cirrhosis with ascites taking furosemide, the fractional excretion of sodium may be higher than one percent. Therefore, administration of diuretics may disturb the electrolyte balance and confuse the clinical settings (4). Additionally, diuretics affect the glucose transport of proximal tubular fluid reabsorption (5). Likewise, urinary sodium in ATN caused by rhabdomyolysis, contrast nephropathy or uric acid nephropathy may be misleading (6). Another limitation for the use of fractional excretion of sodium is due to the reason that sodium reabsorption is in across of nephron; thus in most conditions sodium reabsorption may be influenced by various conditions $(7,8)$.

Lithium may be a more superior and specific parameter than sodium. However, it is parallel to the reabsorption of sodium and water, in the proximal tubule but its reabsorption is limited after passing the proximal tubules (9).

According to the recent data, the average of endogenous serum lithium levels in humans are about $0.0003 \mathrm{mmol} / \mathrm{L}$, which shows low variations $(\sim 0.0001-0.0005)(10,11)$.

Previously it was taught that no endogenous lithium in the human body was existed. The usual total daily lithium intake of nutritional sources has been counted to be 0.05 $\mathrm{mg} / \mathrm{d}(10,11)$. It is detected that tap water comprises a fraction around one tenth of the total lithium intake for the majority of humans.

\section{Objectives}

In this study, the fractional excretion of lithium and sodium is compared to distinguish PRA from ATN.

\section{Patients and Methods \\ Study population}

This cross-sectional study was conducted on 52 acute kidney injury patients consisting of both prerenal azotemia and ATN presenting to the emergency department of Imam Reza hospital (2017-2018). Acute kidney injury was defined as an increase in plasma creatinine greater than $0.3 \mathrm{mg} / \mathrm{dL}$ in 48 hours or more than $50 \%$ of the baseline creatinine within a week or a decrease in urine output of less than $0.5 \mathrm{cc} / \mathrm{kg} / \mathrm{h}$ within 6 hours. Furthermore, 20 healthy humans were also considered as the control group. Afterwards, patients were divided into three groups; patients with ATN, patients with prerenal azotemia and healthy control group.

\section{Definitions}

Blood creatinine, sodium and lithium were checked (for calculating their fractional excretion). Accordingly, kidney ultrasonography was conducted for all patients. PRA group consisted of patients with kidney injury with reduced volume of extracellular fluid, transient hypotension or heart failure, with normal creatinine within 72 hours after the patient's hemodynamic correction, without casts in urine with no evidence of obstruction and also a plasma creatinine of less than $3 \mathrm{mg} / \mathrm{dL}$.

We checked plasma creatinine level 72 hours after the admission too. In addition, urine volume was checked. Accordingly, clinical examination was also conducted. If plasma creatinine was returned to normal range with improvement of urine volume and clinical situation of patients, then, the patient was classified to have PRA.

ATN group consisted of patients with septic shock with long hypotension, rhabdomyolysis or under exposure to contrast agents of nephrotoxic drugs/aminoglycosides or cardiovascular surgery, and with kidney injury, despite the correction of hemodynamic stability of the patient, while the creatinine levels were greater than $3 \mathrm{mg} / \mathrm{dL}$.

If plasma creatinine and urine volume were not returned to suitable range in the following days, and if patient's creatinine returned to normal range after 4-6 weeks, then, they were classified as ATN.

Finally, the healthy controls group consisted of healthy humans which their age and gender were matched with the other two groups.

In this study, the fractional excretion of sodium and lithium were measured. Moreover, sodium, urea and creatinine levels were measured in plasma and urine. Accordingly, the level of lithium was measured using an electrolytic analyzer for plasma and urine.

In addition, fractional excretion of sodium and lithium were calculated according to the following formula;

$\mathrm{FEX}=\mathrm{UX} \times \mathrm{PCr} / \mathrm{PX} \times \mathrm{UCr}$

Where in this relationship FEX, UX and PX are fractional excretion, urinary, plasma sodium and lithium levels, respectively. Accordingly, $\mathrm{PCr}$ and $\mathrm{UCr}$ are referred as plasma and urinary levels of creatinine, respectively. In this study, the ATN patients who were under diuretic therapy, remained on their diuretics 24 hours before the samples were collected. The ATN group was followed between 4 to 6 weeks after diagnosis to ensure the accuracy of diagnosis.

Patients with acute glomerulonephritis, acute tubulointerstitial nephritis and post-renal acute kidney injury and red blood cells and white blood cells and casts in their urine were excluded from this study.

After classifying the patients into, PRA group or ATN group, the fractional excretion of sodium and lithium (FE NA and FE Li) were evaluated.

\section{Ethical issues}

The research followed the tenets of the Declaration of Helsinki. Informed consent was obtained. This study was approved by the Ethics Committee of Mashhad University 
of Medical Sciences (kidney transplant complication research center).

\section{Data analysis}

Descriptive data were expressed as mean and standard deviation (SD). Statistical analysis was conducted using SPSS software, which the $P$ values less than 5\% were considered as statistically significant. For group comparison, student's $t$ test was used. Additionally, for evaluating statistical correlation, Pearson's test was performed. Moreover, sensitivity and specificity in fractional excretion of $\mathrm{Na}$ and $\mathrm{Li}$ for diagnosis PRA from ATN were analyzed with ROC curve.

\section{Results}

In this study, 27 patients in the prerenal azotemia group (21 men and 6 women), 25 patients in ATN group ( 9 females and 16 males) and 20 healthy persons (7 women and 13 men) as the control group were included. In all groups, the proportion of men was higher than that of women. The frequency of gender in all groups had no significant difference $(P=0.49)$ and the groups had the same gender distribution.

Table 1 shows the mean and standard deviation of age and laboratory findings of groups. As expected, the urea and creatinine levels were significantly higher in patients with ATN compared to the control and PRA groups.

The plasma lithium level in both groups of PRA and ATN did not differ. In ATN group, a significant and direct relationship between fractional excretion of sodium and fractional excretion of lithium $(P=0.001)$ was seen. While in PRA group, significant correlation of fractional excretion of sodium with fractional excretion of lithium was not observed $(P=0.26)$.

Area under the curves (AUCs) for fractional excretion of sodium and fractional excretion of lithium were 0.83 $(P=0.001)$ and $0.84(P=0.001)$, respectively. Specificity of fractional excretion of sodium between prerenal azotemia and ATN at the cut off of 2.96 was found to be $68 \%$ and
$75 \%$, respectively. Moreover, the fractional excretion of lithium between prerenal azotemia and ATN at the cut off of 4.17 , was $80 \%$ and $79 \%$, respectively.

\section{Discussion}

In this study, fractional excretion of sodium was not alone shown to be a useful tool in differentiating of ATN from PRA. The previous indices have limitations, especially in cases of administration of diuretics or in the conditions of glycosuria. In this study accuracy, fractional excretion of sodium and lithium, in differentiating prerenal azotemia with ATN was assessed. Both of these indices showed high diagnostic accuracy. However, we found a higher diagnostic value for FE Li in differentiating PRA from ATN. AUC fractional excretion of lithium and sodium for differentiating ATN from prerenal azotemia showed that the diagnostic fractional excretion of lithium was higher than sodium.

In PRA patients, renal sodium and lithium reabsorption were significantly increased and consequently a decrease in fractional excretion of sodium and lithium is predicted. It has been well established that acute kidney injury includes a range of pathologic situations, which differs ranging from a mild impairment of tubular performance with an increase in the capacity of sodium reabsorption to a more severe tubular injury with tubular necrosis. In its natural situation, the secreted lithium ions will reabsorb in proximal tube and to some extent in the loop of Henle, whereas no lithium ion reabsorption takes place in the distal tubule.

This is the basis for the application of the lithium clearance as the index of the proximal tubule excretion fraction. In patients with ATN, a significant positive correlation between fractional excretion of sodium and lithium was detected. While in patients with PRA, no significant correlation between fractional excretion of sodium with lithium was existed. In this study, the cutting point of the highest sensitivity and specificity that differentiates prerenal azotemia from ATN was close to

Table 1. Different measured characteristics for each of the three groups

\begin{tabular}{lccc}
\hline Variable & Prerenal Azotemia & ATN & Control \\
\hline Proportion of patients & 27 & 25 & 20 \\
Gender (female to male ratio) & 0.285 & 0.562 & 0.538 \\
Age (y) & $64.14 \pm 17.92$ & $58.91 \pm 21.59$ & $54.4 \pm 20.12$ \\
Plasma creatinine (mg/dL) & $1.36 \pm 0.25$ & $3.7 \pm 2.5$ & $1.1 \pm 1.04$ \\
Plasma sodium (mg/dL) & $137.48 \pm 4.52$ & $138.5 \pm 8.72$ & $139.06 \pm 3.47$ \\
Plasma lithium (mmol/L) & $0.26 \pm 0.08$ & $0.21 \pm 0.5$ & $0.28 \pm 0.08$ \\
Urine sodium (mmol/L) & $110.4 \pm 0.74$ & $112.31 \pm 57.50$ & $144.46 \pm 0.65$ \\
Urine creatinine (mmol/L) & $57.37 \pm 34.64$ & $41.79 \pm 28.74$ & $89.3 \pm 51.6$ \\
Urine lithium (mmol/L) & $0.33 \pm 0.21$ & $0.21 \pm 0.13$ & 0.7 \\
FE Na \% & $2.09 \pm 1.36$ & $12.44 \pm 17.55$ & $0.51 \pm 0.31$ \\
FE Li \% & $3.41 \pm 2.81$ & $35.52 \pm 107.08$ & $1.59 \pm 1.49$ \\
\hline
\end{tabular}


$3 \%$. While generally fractional excretion of sodium below one percent for the patient suggests prerenal azotemia. The values between 1 to 2 percent may be seen in both cases. Values of more than $2 \%$ were suggested in ATN patients. In our study, a possible cause for this difference is due to the patients with more than $1 \%$ of fractional excretion having prerenal azotemia were not excluded. The most important cause of prerenal azotemia is the administration of diuretics. The diuretics that affect the loop of Henle influence the transfer of sodium in the loop. In high doses, the proximal tube is affected, too. Thiazide diuretics inhibit the reabsorption of sodium in distal tubule (4).

In addition to using diuretics, other scenarios resulted in prerenal azotemia and increased fractional excretion of sodium. The main reseaon is the decline in body volume caused by vomiting or nasogastric aspiration with increased fractional excretion of sodium, bringing about bicarbonaturia. A limitation on the use of fractional excretion of sodium is the fact that sodium reabsorption is conducted throughout the nephron (7). In the circumstance of hypovolemia, increased aldosterone and renal reabsorption of sodium and water in the proximal tubules.

The fractional excretion of sodium, fewer than one percent, is not always abnormal, but is a natural reaction of sodium homeostasis in patients with a low or moderate sodium intake in diet. Therefore, the use of fractional excretion of sodium alone will not be accurate always, but with the simultaneous use of this biomarker and fractional excretion of lithium, ATN can be differentiated from PRA.

The ability of a material to provide a valid estimate of proximal tubule reabsorption is based on two assumptions. First, the concentration of tubular fluid into the plasma ratio is closer. Second, it must not be reabsorbed in the proximal tubule (8). Therefore, lithium may be a more specific indicator for proximal tubule, due to the parallel reabsorption of sodium and water in the proximal tubule, but reabsorption is limited after the other proximal tubule (9).

Thiazides do not seem to directly affect the fractional excretion of lithium but have the possibility to reabsorb lithium, which is particularly controversial in the thick limp of the Henle loop of patients treated with diuretics or reduce the volume in the distal tubules. However, lithium clearance, up to this time, can be a good approximation of the proximal tubular function (12).

In the study of Pepin et al, sensitivity and specificity of FENa, in the diagnosis of acute kidney injury was reported to be $78 \%$ and $75 \%$, respectively (13). In a study by Steinhauslin and colleagues, fractional excretion of sodium, uric acid and lithium was conducted in 46 patients (28 patients had prerenal azotemia and 18 of them had ATN). They found that clinical diagnosis of patients, when matched with fractional excretion of lithium was $78 \%$, while the fractional excretion of sodium and uric acid were matched in $63 \%$ and $50 \%$ of patients, respectively. In addition, the sensitivity of fractional excretion of lithium in the determination of hemodynamic insufficiency in acute renal failure was higher than that for sodium and uric acid. Moreover, the reduced fractional excretion of lithium, sodium and uric acid in patients with prerenal acute kidney injury were $93 \%, 75 \%$ and $68 \%$, respectively (14). While, in our study, the sensitivity and specificity of fractional excretion of sodium between PRA and ATN, was $68 \%$ and $75 \%$, respectively. Additionally, the sensitivity and specificity of fractional excretion of lithium were $80 \%$ and $79 \%$, respectively.

Although the study by Steinhauslin et al showed that fractional excretion of lithium is preferred (14), its application is not common, probably because the test is expensive. Besides there is the impression that the administration of additional lithium is required for testing.

Accordingly, in the study by Steinhauslin (14), the proposed cut-off point, especially in the case of fractional excretion of lithium comparing to our study was different, which can be due to the differences in the measurement procedures. In the study by Steinhauslin, the spectrophotometric method was used, while in our study, urine and plasma levels of lithium were measured by the electrolytic analyzer.

\section{Conclusion}

This study showed that fractional excretion both lithium and sodium have high AUC and high diagnostic accuracy for diagnosis, but the diagnostic test for fractional excretion of lithium is more accurate than sodium. We also found that fractional excretion of lithium was superior to $\mathrm{FE} \mathrm{Na}$ and uric acid.

\section{Limitations of the study}

Our study was conducted on a limited proportion of patients and additional studies must be carried out by larger samples on this subject.

\section{Acknowledgements}

The authors would like to thank the Research Council of Mashhad University of Medical Sciences for supporting the study financially.

\section{Authors' contribution}

FS: data collection and manuscript drafting, AAZ: study design, statistical analysis. ZL: data collection and manuscript reviewing. TZ, FM and FS contributed to the development of the study. All authors read and signed the final paper.

\section{Conflicts of interest}

The authors declare no conflict of interest.

Ethical considerations

Ethical issues (including plagiarism, data fabrication, 
double publication) have been completely observed by the authors.

\section{Funding/Support}

This article is taken from the nephrology fellowship thesis of Farnaz Sahihi. This study was supported by a grant (Grant \# 930212) from the Research Center of Mashhad University of Medical Sciences kidney transplant complications.

\section{References}

1. Vaara ST, Pettilä V, Kaukonen KM, Bendel S, Korhonen AM, Bellomo R, et al. Finnish Acute Kidney Injury Study Group. The attributable mortality of acute kidney injury: a sequentially matched analysis. Crit Care Med.2014;42:87885. doi: 10.1097/CCM.0000000000000045.

2. Gotfried J, Wiesen J, Raina R, Nally Jr. Finding the cause of acute kidney injury: which index of fractional excretion is better? Cleve Clin J Med.2012;79:121-6. doi: 10.3949/ ccjm.79a.11030.

3. Nejat M, Pickering JW, Devarajan P, Bonventre JV, Edelstein CL, Walker RJ, et al. Some biomarkers of acute kidney injury are increased in pre-renal acute injury. Kidney Int. 2012;17:1254-62. doi: 10.1038/ki.2012.23.

4. Dewitte A, Biais M, Petit L, Cochard JF, Hilbert G, Combe $\mathrm{C}$, et al .Fractional excretion of urea as a diagnostic index in acute kidney injury in intensive care patients. J Crit Care. 2012;27:505-10. doi: 10.1016/j.jcrc.2012.02.018.

5. Pons B, Lautrette A, Oziel J, Dellamonica J, Vermesch R, Ezingeard E, et al. Diagnostic accuracy of early urinary index changes in differentiating transient from persistent acute kidney injury in critically ill patients: multicenter cohort study.Crit Care.2013;17:56. doi:10.1186/cc12582.

6. Wlodzimirow KA, Abu-Hanna A, Royakkers AA, Spronk PE, Hofstra LS, Kuiper MA, et al. Transient versus persistent acute kidney injury and the diagnostic performance of fractional excretion of urea in critically Ill Patients.Nephron
Clin Pract. 2014;126:8-13. doi: 10.1159/000357678.

7. 7.Vanmassenhove J, Glorieux G, Hoste E, Dhondt A, Vanholder R, Van Biesen W. Urinary output and fractional excretion of sodium and urea as indicators of transient versus intrinsic acute kidney injury during early sepsis. Crit Care. 2013;17:234. doi: 10.1186/cc13057.

8. Jiao M, Ren F, Zhou L, Duan Z, Zhao C. The protective role of peroxisome proliferator activated receptors- $\alpha$ and pathomechanism in Dgalactosamine/lipopolysaccharideinduced acute liver failure in rats. Zhonghua Nei Ke Za Zhi. 2014;53:730-4.

9. Zhao J, Gao P, Wu S, Zhu D. Determination of trace lithium in human urine by electrothermal atomic absorption spectrometry using nitric acid as a chemical modifier to eliminate the interference of chloride. Anal Sci. 2009;25: 639-43. doi: 10.2116/analsci.25.639.

10. Anon, Second French Total Diet Study (TDS 2): Report 1 Inorganic contaminants, minerals, and persistent organic pollutants, mycotoxins a phytoestrogen. 2012

11. Noël L, Chekri R, Millour S, Vastel C, Kadar A, Sirot V, et al. $\mathrm{Li}, \mathrm{Cr}, \mathrm{Mn}, \mathrm{Co}, \mathrm{Ni}, \mathrm{Cu}, \mathrm{Zn}$, Se and Mo levels in foodstuffs from the Second French TDS. Food Chem. 2012;132:15021513. doi: 10.1016/j.foodchem.2011.12.009.

12. Sinke AP, Kortenoeven ML, de Groot T, Baumgarten R, Devuyst O, Wetzels JF, et al. Hydrochlorothiazide attenuates Lithium-induced nephrogenic diabetes insipidus independently of the sodium-chloride cotransporter. Am J Physiol Renal Physiol. 2014;306:525-33. doi: 10.1152/ ajprenal.00617.2013.

13. Pépin MN, Bouchard J, Legault L, Ethier J. Diagnostic performance of fractional excretion of urea and fractional excretion of sodium in the evaluations of patients with acute kidney injury with or without diuretic treatment. Am J Kidney Dis. 2007;50:566-73. doi:10.1053/j.ajkd.2007.07.001

14. Steinhäuslin F, Burnier M, Magnin JL, Munafo A, Buclin T, Diezi J, et al. Fractional excretion of trace Lithium and uric acid in acute renal failure. J Am Soc Nephrol.1994;4:1429-37.

Copyright ( $\odot 2020$ The Author(s); Published by Nickan Research Institute. This is an open-access article distributed under the terms of the Creative Commons Attribution License (http://creativecommons.org/licenses/by/4.0), which permits unrestricted use, distribution, and reproduction in any medium, provided the original work is properly cited. 